\title{
Stochastic calculation of the QCD Dirac operator spectrum with Mobius domain-wall fermion
}

\section{G. Cossu}

High Energy Accelerator Research Organization (KEK), Tsukuba 305-0801, Japan

\section{H. Fukaya}

Department of Physics, Osaka University, Toyonaka 560-0043, Japan

\section{S. Hashimoto*}

High Energy Accelerator Research Organization (KEK), Tsukuba 305-0801, Japan and School of High Energy Accelerator Science, SOKENDAI (The Graduate University for Advanced Studies), Tsukuba 305-0801, Japan

E-mail: shoji.hashimotodkek.jp

\section{T. Kaneko}

High Energy Accelerator Research Organization (KEK), Tsukuba 305-0801, Japan and SOKENDAI (The Graduate University for Advanced Studies), Tsukuba 305-0801, Japan

\section{J. Noaki}

High Energy Accelerator Research Organization (KEK), Tsukuba 305-0801, Japan

\begin{abstract}
We calculate the spectral function of the QCD Dirac operator using the four-dimensional effective operator constructed from the Mobius domain-wall implementation. We utilize the eigenvalue filtering technique combined with the stochastic estimate of the mode number. The spectrum in the entire eigenvalue range is obtained with a single set of measurements. Results on 2+1-flavor ensembles with Mobius domain-wall sea quarks at lattice spacing $\sim 0.08 \mathrm{fm}$ are shown.
\end{abstract}

The 33rd International Symposium on Lattice Field Theory

14 - 18 July 2015

Kobe International Conference Center, Kobe, Japan*

\footnotetext{
${ }^{*}$ Speaker.
} 


\section{Introduction}

The eigenvalue density (or spectral function) of the Dirac operator

$$
\rho(\lambda)=\frac{1}{V}\left\langle\sum_{i} \delta\left(\lambda-\lambda_{i}\right)\right\rangle
$$

provides a probe of the spontaneous chiral symmetry breaking in QCD through the Banks-Casher relation $\rho(0)=\Sigma / \pi[\mathbb{U}$ ], where $\Sigma$ denotes the chiral condensate $\Sigma=-\langle\bar{q} q\rangle$ in the thermodynamical limit. The functional form of $\rho(\lambda)$ at small $\lambda$ is computed by one-loop chiral perturbation theory in the $p$-regime [ [] ] and in the mixed regime [B], but the value of $\Sigma$ is to be determined by nonperturbative QCD calculations.

The most direct way of obtaining the eigenvalue density in lattice QCD is to calculate the individual low-lying eigenvalues and to count the number of them falling in a region suffciently close to zero. This method was adopted in our previous works to extract the chiral condensate in 2+1-flavor QCD [四, 回] using the overlap-Dirac operator. For larger volumes, however, it becomes computationally more demanding because of the cost and memory requirement of the Lanczos-type algorithms.

An alternative way is to stochasitically estimate the number of eigenvalues below some threshold. It was first implemented in [囫] for this particular problem. In this work we introduce a variant of this method to calculate the spectral function. Namely, we utilize the Chebyshev filtering technique combined with a stochastic estimate of the mode number. As described in the next section, the method is more flexible and can be used to calculate the whole spectrum at once. We use the lattice ensembles generated with $2+1$ flavors of the Mobius domain-wall fermion at a lattice spacing $a \simeq 0.08 \mathrm{fm}$.

\section{Chebyshev filetering}

One can evaluate the number of eigenvalues in an interval $[a, b]$ of a hermitian matrix $A$, which is supposed to be $D^{\dagger} D$ of any lattice Dirac operator $D$, as

$$
n[a, b]=\frac{1}{N_{v}} \sum_{k=1}^{N_{v}} \xi_{k}^{\dagger} h(A) \xi_{k}
$$

with Gaussian random vectors $\xi_{k}$, which has a normalization $\left(1 / N_{v}\right) \sum_{k=1}^{N_{v}} \xi_{k}^{\dagger} \xi_{k}=12 \mathrm{~V}$ in the limit of large $N_{v}$ the number of random vectors. $h(A)$ is a function of matrix $A$ that works as a filter of eigenvalues. Without $h(A)$, (2. returning 1 in the range $[a, b]$ and 0 elsewhere, we may stochastically count the number of modes in that interval. The statistical error is given by a square-root of the mode number in $[a, b]$; when the number of eigenvalues in the range $[a, b]$ is sufficiently large, $N_{v}=1$ could already give a precise estimate.

One can use the Chebyshev polynomial $T_{j}(x)$ to approximate the filter $h(x)$ :

$$
h(x)=\sum_{j=0}^{p} g_{j}^{p} \gamma_{j} T_{j}(x) .
$$




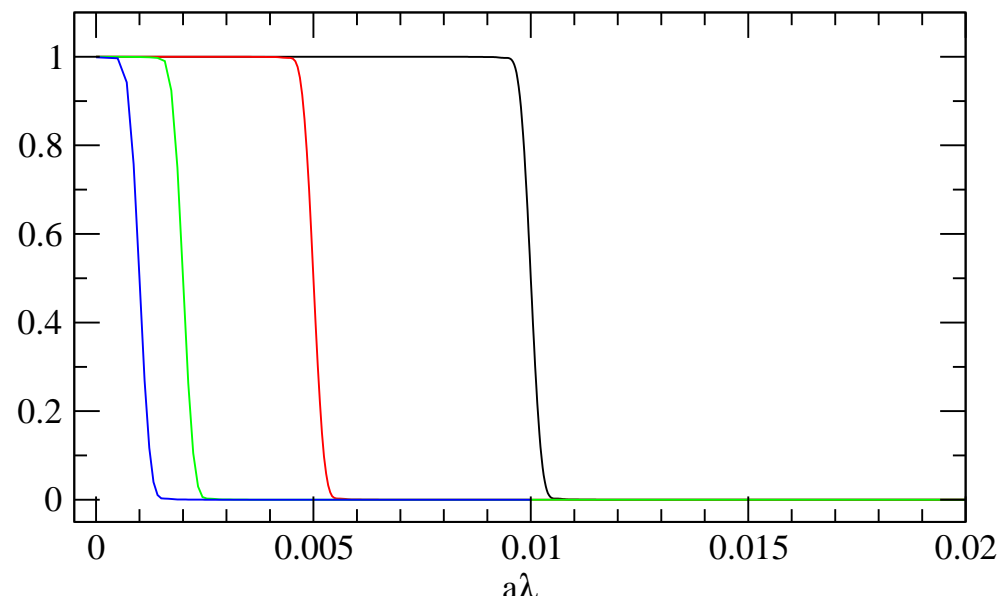

Figure 1: Step function approximation given by the Chebyshev polynomial at order $p=8000$. Typical results for the interval $[0, \delta]=0.01,0.005,0.002$ and 0.001 are shown from right to left.

The coefficients $\gamma_{j}$ and $g_{j}^{p}$ are known numbers fixed once the interval $[a, b]$ is given. The conventional Chebyshev minmax approximation is obtained with $\gamma_{j}$, while the Jackson stabilization factor $g_{j}^{p}$ is introduced to suppress the oscillation typical in the Chebyshev expansion [ $[$ ] In order for the Chebyshev approximation to work, the whole eigenvalues of $A$ have to be in the range $[-1,1]$.

After the ensemble average (over gauge configurations) one obtains

$$
\bar{n}[a, b]=\frac{1}{N_{v}} \sum_{k=1}^{N_{v}}\left[\sum_{j=0}^{p} g_{j}^{p} \gamma_{j}\left\langle\xi_{k}^{\dagger} T_{j}(A) \xi_{k}\right\rangle\right] .
$$

An important observation is that once the stochastic estimates of $\left\langle\xi_{k}^{\dagger} T_{j}(A) \xi_{k}\right\rangle$ are calculated for each $j$ the eigenvalue count in any interval $[a, b]$ can be obtained by combining them with the corresponding coefficients $g_{j}^{p} \gamma_{j}$. Namely, the interval can be adjusted afterwards, independent of the costly calculation of the polynomial of the matrix $A$. Details of the method are found in [ $[$ ] .

The Chebyshev polynomial can be easily constructed using the recurrence formula

$$
T_{0}(x)=1, \quad T_{1}(x)=x, \quad T_{j}(x)=2 x T_{j-1}(x)-T_{j-2}(x) .
$$

One can also use the relations $T_{2 n-1}(x)=2 T_{n-1}(x) T_{n}(x)-T_{1}(x)$ and $T_{2 n}(x)=2 T_{n}^{2}(x)-T_{0}(x)$, in order to reduce the numerical efforts. With infinitely large $p$ the filtering function $h(x)$ is exactly reproduced; at finite $p$, the approximating function is smeared around the borders $a$ and $b$, leading to a systematic error.

For the four-dimensional effective Dirac operator of domain-wall fermion, the eigenvalues of

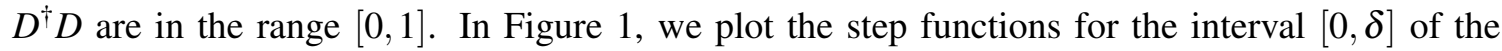
eigenvalue of $|D|$ with $\delta=0.01,0.005,0.002$ and 0.001 . (For the correspondence between the eigenvalues of $D^{\dagger} D$ and those of $|D|$, see below.) The polynomial order is fixed to $p=8000$. One can see that the step function is well approximated away from the boundary. Near the boundary, the edge is rounded off. Its effect is relatively more important for smaller $\delta$. The error estimated for the area, which has to be $\delta$, is $0.8 \%$ for $\delta=0.01$ and $1.5 \%$ for $\delta=0.005$, scaling as $1 / \delta$. 


\section{Lattice calculation}

The JLQCD collaboration has generated a new set of ensembles of 2+1-flavor QCD with Mobius domain-wall fermion for sea quarks. It aims at achieving good chiral symmetry, i.e. the residual mass is order $1 \mathrm{MeV}$ or smaller. Three lattice spacings are chosen as $1 / a=2.45,3.61$ and $4.50 \mathrm{GeV}$, which allow well controlled continuum extrapolation even including charm quarks as valence quarks. The up and down quark masses correspond to the pion mass $M_{\pi}$ of 230, 300, 400 and $500 \mathrm{MeV}$; two strange quark masses are chosen such that they sandwich the physical value. Lattice volume is $32^{3} \times 64,48^{3} \times 96$ and $64^{3} \times 128$, depending on the lattice spacing, and the physical volume satisfies the nominal condition $M_{\pi} L>4$. This set of ensembles have been used

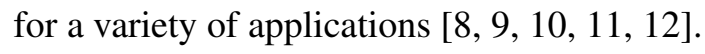

In this preliminary work, we use the coarse lattice $(1 / a=2.45 \mathrm{GeV})$ of size $32^{3} \times 64$, out of the above mentioned ensembles. The number of configurations is 50 for each ensemble, taken out of $10,000 \mathrm{HMC}$ trajectories. The number $N_{v}$ of the Gaussian noise vector $\xi_{k}$ is 1 . The up and down quark masses in the lattice unit are $a m_{u d}=0.019,0.012,0.007$ and 0.0035 .

We calculate the eigenvalue density of the hermitian operator $D^{(4) \dagger} D^{(4)}$ made of the fourdimensional (4D) effective operator

$$
D^{(4)}=\left[P^{-1}\left(D^{(5)}(m=1)\right)^{-1} D^{(5)}(m=0) P\right]_{11} .
$$

Here, $D^{(5)}(m)$ represents the five-dimensional (5D) Mobius domain-wall operator with mass $m$. For the eigenvalue count we took $m=0$, i.e. the massless Dirac operator. The $4 \mathrm{D}$ effective operator is constructed by multiplying the inverse of the Pauli-Villas operator $(m=1)$ and taking the $4 \mathrm{D}$ surfaces (represented by the subscript "11") appropriately projected onto left- and right-handed modes by a projection operator $P$. See, for instance, [एँ3] for more details.

For each application of $D^{(4)}$ on $4 \mathrm{D}$ vectors, we have to calculate the inverse of the PauliVillars operator, for which the conjugate gradient iteration of order 40-50 is involved. Although the inversion is much less expensive than the calculation of light quark propagator, the total numerical cost is substantial because we have to multiply $D^{(4) \dagger} D^{(4)} p$-times. ( $p=8000$ in this analysis.)

The eigenvalues of $D^{(4) \dagger} D^{(4)}$ are in the region $[0,1]$, and we rescale the operator as $A=$ $2 D^{(4) \dagger} D^{(4)}-1$ to match the region of the Chebyshev approximation. Since the effective 4D operator satisfies the Gisparg-Wilson relation very precisely, we assume that eigenvalues of $D^{(4)}$ lie on a circle in the complex plane. In the following, the eigenvalue $\lambda$ stands for that projected onto the imaginary axis as $\lambda=\sqrt{\lambda_{D^{(4) \dagger} D^{(4)}} /\left(1-\lambda_{D^{(4) \dagger} D^{(4)}}\right)}$.

Figure $\square$ shows the eigenvalue spectrum for the whole range of $\lambda$ in the lattice unit. Both axes are in a logarithmic scale. For each bin of $[a, b]$, it is constructed as $\rho(\lambda ; \delta)=(1 / 2 V) \bar{n}[a, b] / \delta$ with a bin size $\delta$. (Therefore, it satisfies $\lambda=\sqrt{a /(1-a)}$ and $\lambda+\delta=\sqrt{b /(1-b)}$.)

One can clearly see that the number of eigenvalues increases toward higher $\lambda$ and saturate at some point of $O(1)$ due to the discretization effect, which should otherwise behave like $\sim \lambda^{3}$ for asymptotically large $\lambda$. There is no visible quark mass dependence in this region. On the lowest end, it approaches a constant corresponding to $\rho(0)$, from which one extracts the chiral condensate.

The same data are plotted in Figure $[$ in a linear scale. The individual bin has a width of $\delta=$ 0.005. With this binsize, the systematic error due to the Chebyshev approximation is well below the statistical error. 


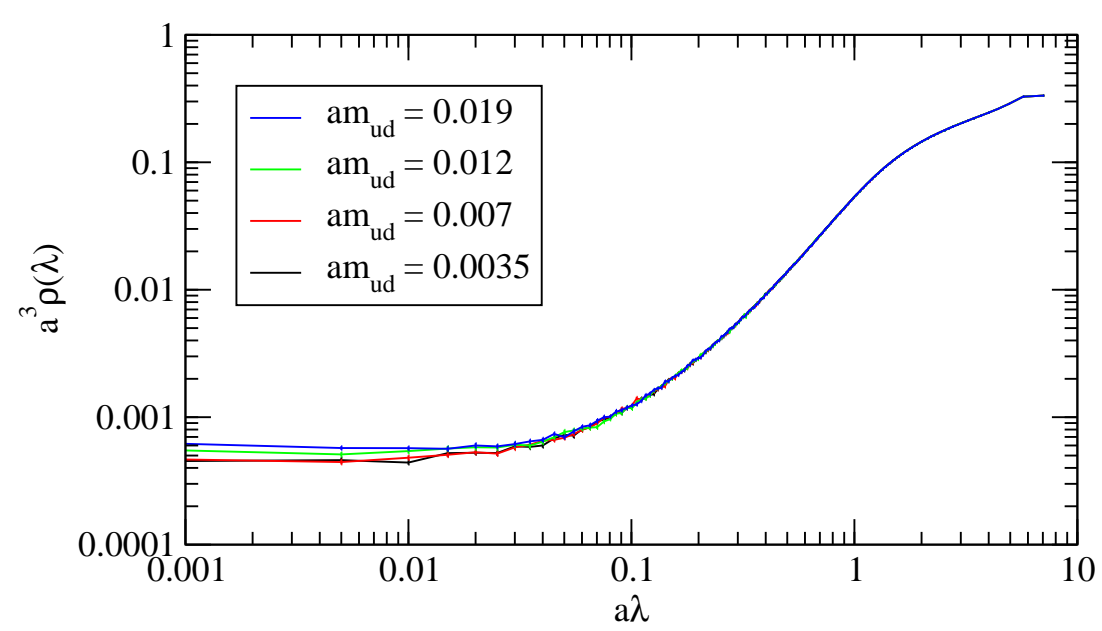

Figure 2: Spectral function in a logarithmic scale. The lattice data are plotted for four values of up and down quark masses.

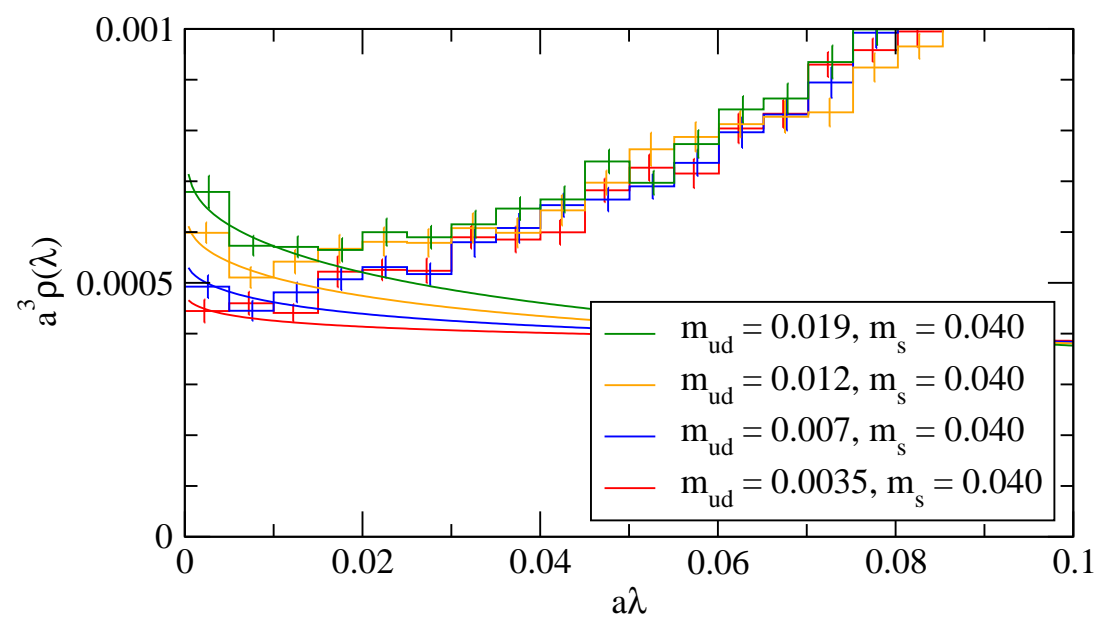

Figure 3: Spectral function in a linear scale. The binsize is taken as $\delta=0.005$. The lattice data are plotted for four values of up and down quark masses (histogram with four different colors). Curves are those of one-loop chiral perturbation theory.

\section{Analysis using $\chi$ PT formula}

Figure [ 3 shows a clear dependence of $\rho(0)$ on the sea up and down quark mass $m_{u d}$. Namely, $\rho(0)$ gets lower for smaller $m_{u d}$. Furthermore, a peak develops near $\lambda=0$ for heavier sea quarks. Qualitatively, it is understood as the effect that the fermion determinant is no longer active below $\lambda \lesssim m_{u d}$ to suppress the near-zero modes. More near-zero eigenvalues may then survive for larger $m_{u d}$.

In order to obtain the chiral condensate $\Sigma$, one has to take the thermodynamical limit, i.e. the infinite volume limit and then the massless quark limit. The order of the limits is crucial; $\rho(0)$ vanishes in the massless limit on any finite volumes. Fortunately, such volume and mass dependences are well understood in chiral perturbation theory $(\chi \mathrm{PT})$, and we may identify the volume beyond which the system is effectively in the large volume limit. All our lattices satisfy 


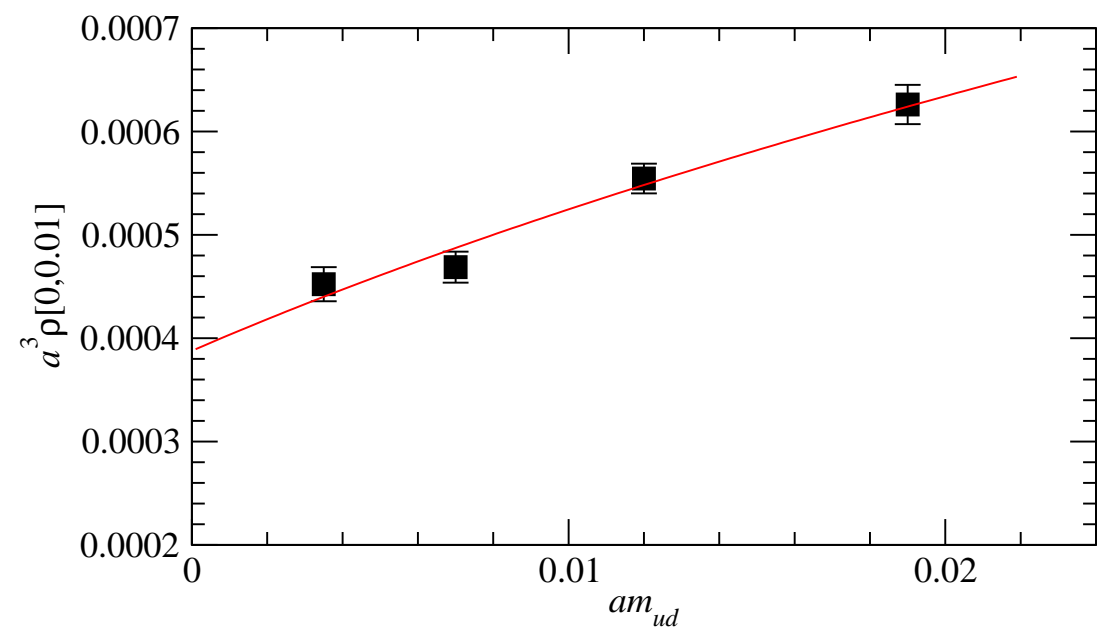

Figure 4: Chiral extrapolation of the partially integrated spectral function $\rho[0, \delta]$ with $\delta=0.01$. The oneloop $\chi$ PT curve at $N_{f}=2$ is shown together with the lattice data (black square).

that condition, and we use the $p$-regime $\chi \mathrm{PT}$ formula in the following analysis.

One-loop formula for $N_{f}=2$ is available in []]. It is written in terms of the leading order low-energy constants (LEC) $\Sigma$ and $F$ as well as the next-to-leading order LEC $L_{6}$. The pion decay constant controls the size of the next-to-leading order corrections. In this preliminary analysis, we fix it to a nominal value $F=90 \mathrm{MeV}$.

We fit the value of $\bar{\rho}[0, \delta]=(1 / \delta) \int_{0}^{\delta} d \lambda \rho(\lambda)$ with the one-loop $\chi$ PT expression with $\delta=$ 0.01 . Namely, both the lattice data and one-loop $\chi \mathrm{PT}$ are integrated in the same region. This value of $\delta$ corresponds to the scale of pion mass of $\delta \Sigma / F^{2} \simeq 300 \mathrm{MeV}$, for which one expects that one-loop $\chi$ PT converges reasonably well.

Chiral extrapolation of $\rho[0,0.01]$ is shown in Figure 1 . The one-loop $\chi$ PT curve shows a slight curvature due to the chiral logarithm. The fit yields $\Sigma^{1 / 3}=262.0(1.7) \mathrm{MeV}$ and $L_{6}=0.00031$ (7) with $\chi^{2} /$ dof $=1.13$.

Renormalizing to the $\overline{\mathrm{MS}}$ scheme at $2 \mathrm{GeV}$, we obtain $[\Sigma(2 \mathrm{GeV})]^{1 / 3}=260.0(1.7) \mathrm{MeV}$, where we use the renormalization factor $Z_{S}(2 \mathrm{GeV})$ determined from the analysis of short-distance current correlator [ए]]].

Probably because the strange quark is too heavy to apply one-loop $\chi \mathrm{PT}$, a fit with the $2+1$ flavor $\chi$ PT formula failed to reproduce the lattice data.

\section{Discussions}

The Chebyshev filtering technique allows precise evaluation of the eigenvalue count in a sufficiently small bin to calculate the eigenvalue spectrum. The method is especially suitable for the 4D effective operator of the domain-wall fermion since the eigenvalue of $D^{(4) \dagger} D^{(4)}$ is limited in $[0,1]$. For the Wilson fermion, the range is $[0,64]$ (in the free theory), and one needs much higher order polynomial to obtain the same precision. This would nearly compensate the numerical effort to construct the (expensive) 4D effective Dirac operator from domain-wall fermion. 
This preliminary analysis has been done using partial data out of the full data set at three lattice spacings and various sea quark masses. We plan to include the data on finer lattices that allow us to extrapolate to the continuum limit.

We are grateful to Julius Kuti for private communications on the technique employed in this work. Their own work was also presented at this conference. Numerical calculation was performed on the Blue Gene/Q supercomputer at High Energy Accelerator Research Organization (KEK) under a support of its Large Scale Simulation Program (No. 14/15-10). The code set Iroiro++ [प4], which is highly optimized for Blue Gene/Q, is used. This work is supported in part by JSPS KAKENHI Grant Number 26247043.

\section{References}

[1] T. Banks and A. Casher, Nucl. Phys. B 169, 103 (1980). doi:10.1016/0550-3213(80)90255-2

[2] A. V. Smilga and J. Stern, Phys. Lett. B 318, 531 (1993). doi:10.1016/0370-2693(93)91551-W

[3] P. H. Damgaard and H. Fukaya, JHEP 0901, 052 (2009) doi:10.1088/1126-6708/2009/01/052 [arXiv:0812.2797 [hep-lat]].

[4] H. Fukaya et al. [JLQCD Collaboration], Phys. Rev. Lett. 104, 122002 (2010) [Phys. Rev. Lett. 105, 159901 (2010)] doi:10.1103/PhysRevLett.104.122002, 10.1103/PhysRevLett.105.159901 [arXiv:0911.5555 [hep-lat]].

[5] H. Fukaya et al. [JLQCD and TWQCD Collaborations], Phys. Rev. D 83, 074501 (2011) doi:10.1103/PhysRevD.83.074501 [arXiv:1012.4052 [hep-lat]].

[6] L. Giusti and M. Luscher, JHEP 0903, 013 (2009) doi:10.1088/1126-6708/2009/03/013 [arXiv:0812.3638 [hep-lat]].

[7] E. Di Napoli, E. Polizzi, Y. Saad, arXiv:1308.4275 [cs.NA].

[8] J. Noaki et al. [JLQCD Collaboration], PoS LATTICE 2014, 069 (2014).

[9] H. Fukaya et al. [JLQCD Collaboration], arXiv:1509.00944 [hep-lat].

[10] M. Tomii et al. [JLQCD Collaboration], arXiv:1511.09170 [hep-lat].

[11] K. Nakayama, B. Fahy and S. Hashimoto, arXiv:1511.09163 [hep-lat].

[12] B. Fahy et al. [JLQCD collaboration], PoS LATTICE 2015, 074 (2016).

[13] P. A. Boyle [UKQCD Collaboration], PoS LATTICE 2014, 087 (2015).

[14] G. Cossu, J. Noaki, S. Hashimoto, T. Kaneko, H. Fukaya, P. A. Boyle and J. Doi, arXiv:1311.0084 [hep-lat]. 